\title{
Heart Rate Analysis Applied to Beginners Electrician Linemen of Energy Distribution Networks Who Ascend and Descend from the Posts
}

\author{
Ernesto A. Abril ${ }^{1}$, Ernesto M. Abril ${ }^{2}$, Idalia Pacheco ${ }^{2}$, Leonardo M. Abril ${ }^{2}$ and Christian P. Abril ${ }^{3}$ \\ ${ }^{1}$ Departamento de Eléctrica y Electrónica, Universidad de las Fuerzas Armadas ESPE, Sangolquí-Ecuador \\ ${ }^{2}$ Facultad de Ciencias de la Ingeniería y Aplicadas, Universidad Técnica de Cotopaxi, Latacunga-Ecuador. \\ ${ }^{3}$ Departamento de Ciencias Económicas Administrativas y de Comercio, Universidad de las Fuerzas Armadas ESPE, Sangolquí-Ecuador
}

\begin{abstract}
The investigation uses a group of people: an expert and five inexperienced ones in the ascent to the posts of the electric power distribution networks that are 12 meters high. The analysis consisted in taking data related to the heartbeat given by the physical effort made to raise and lower poles, presenting a map of heat, generating a stress caused by the fear of heights and the possibility of slipping or falling from the structures that they are installing, resulting in a very unstable heart rate. The analysis considers different circumstances, such as the correlation of the increase of the heart rate with the effort made by the worker, and the heart rate that increases with the fear or stress of working at height is determined. Should be considered the cardiac pulse that the worker has when descending from the post. The heart rate is variable in all the people analyzed, after a physical effort the heart rate is unpredictable for several people.
\end{abstract}

\section{Introduction}

At present, exists a few studies of cardiology phenomenon that occur in workers who work at high altitudes on poles and electrical towers. The nerves of the person produces a danger given by the heart rate of each electrician. At work height, is important to add the danger of electric shock due to medium voltage work. Even with disconnected system networks, exists the possibility of atmospheric electric discharges, or by the presence of energy caused by manipulations of emergency generators of users who can connect their generators to the networks of the electric company, actions that would compromise the lives of people by having an electric shock through their bodies.

The heartbeat data serve enormously in very high risk jobs, where the worker has extreme anxiety, which can have a mistake in the lines of a thousand volts or more. The great variability of the rhythm of the heart gives us an anxiety, presenting curves with very steep slopes.

To serve the population with electric power, the electrician lineman must manipulate equipment, materials and tools in transmission, sub transmission and distribution lines, which conduct energy from generators to consumers. The workers dedicated to these activities must undergo an analysis of their heart rate, for which the companies that provide these services must have manuals that demand and regulate these control activities. Workers in these activities must not exceed the maximum pulse allowed.
The analysis make emphasis in the physical and psychological behavior of electricians. Recommendations are included before, during and after the work tests, obtaining favorable results for the group of electricians.

The participants submitted to these tests should not smoke or drink alcohol; they should be in good physical condition of their body.

There are researches, which indicate that in moderate exercises, there will be a reserve of heartbeats from $40 \%$ to $50 \%$, but with highly demanded exercises it will be $60 \%$ to $70 \%$, always considering the established in the step test that determines HRmax is the maximum heart rate and it depends on the age of the person. [1]

$$
\text { HRmax }=220-\text { age years }
$$

Thanks to the electrocardiograms and a series of equipment that analyzes the heartbeat, for several years the cardiac pulse has been studied, registering its frequency and variability.

Psychologically, the correlation of stress and anxiety with the heart rate is established. [2]

This study was performed to evaluate 118 subjects (athlete's runners, weight lifters, with control group of healthy subjects sedentary) who have performed stress with treadmill test to evaluate the vagal reserve, as the grade of acceleration of the heart rate (HR) after the 10 second of starting the exercise and the $\Delta 1$ and $\Delta 2$ respectively, Transtoracic echocardiography was performed to evaluate diameters and ventricular volumes and diastolic function to determine the patterns of 
ventricular filling. The sample was divided into three groups: Group I Sedentary, Group II Runners, and the Group III Weight Lifters. Group II was those with vagal predominance in the test with a $\Delta 1 \mathrm{de} \mathrm{m}=36 \pm 14 \mathrm{lat} / \mathrm{min}$ and $\Delta 2 \mathrm{de} \mathrm{m}=58 \pm 15 \mathrm{lat} / \mathrm{min}$ (p0.0001 and expressed left ventricular (LV) geometric patterns given by higher LV diameter and higher volumes indexed $(\mathrm{m}=63 \pm 82 \mathrm{~mm}) \mathrm{p}$ 0.0001 in relation to the group I and III. On the other hand, the weight lifters expressed the less HR recovery after the exercise and the higher wall thickness and higher relation $\mathrm{E} / \mathrm{e}(\mathrm{m}=8 \pm 3)$. $\mathrm{p} 0.0001$. This study allows us to affirm on the vagal predominance and less stiffness, was observed in endurance athletes, compared to athlete's static and also allows opening a light to the explanation of the possible reasons to elucidate this phenomenon. [3]

The experimentation of the research was carried out in the city of Ambato, province of Tungurahua, country of Ecuador in South America. The event was attended by people who live in different parts of the country, therefore, from different heights above sea level as they were from the city of Puyo which is 1000 meters above sea level, from the city of Baños which is at 1700 meters above sea level and Ambato itself which is 2700 meters above sea level. Mainly with an aspiring lineman from the city of Puyo, pressure was inconvenient because only by fear of climbing the post when the climbers surrendered to be placed on the feet and had 130 beats per minute, as they approached the post their pulse rose at 135 in a minute, when ascending approximately 1 meter the pulsations were accelerated to 155 in a minute, when climbing up to 2 meters their pulsations reached 170 in minute, which forced to suspend the ascent in the post and lower it, to which the lineman said that was no longer moving forward.

These tests should be applied to all electricians' linemen, mainly to beginners or novices to have no misfortunes to regret.

It is necessary to consider that for the work which is in heights, the equipment of the workers is extremely heavy consisting of climbers, harness, safety belts, helmets, to more of shoes and own clothes of electricians. Tools and equipment that must transport and can reach 32 Kilograms, all this complicates people of extreme weight exceeding 130 kilograms, and this people would not participate in the tests for not being able to work in distribution networks. [4]

The requirement is also directed to the prohibition of smoking during all working time, even worse to be with alcohol in the blood, cannot be allowed to use cell phones, but only radio communication equipment. [5]

\section{Development}

\subsection{Thermal map of the electrician lineman's body.}

Preliminarily, we had the thermal configuration of the body; we found increases in temperature in the areas of greatest effort. Figure 1.

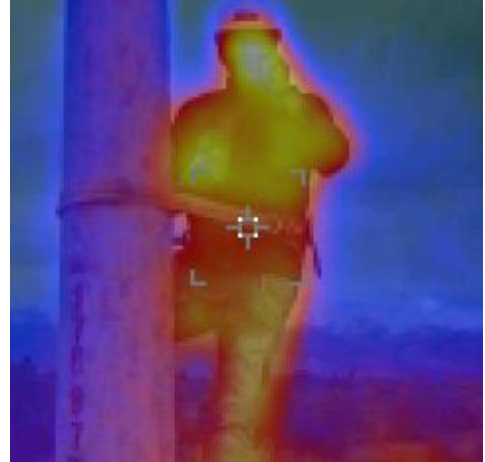

Figure 1. Start climbing.

As the electrician continues to ascend the post, the temperature has an evolution. There is a good presence of heat in the legs, arms and chest, Figure 2.

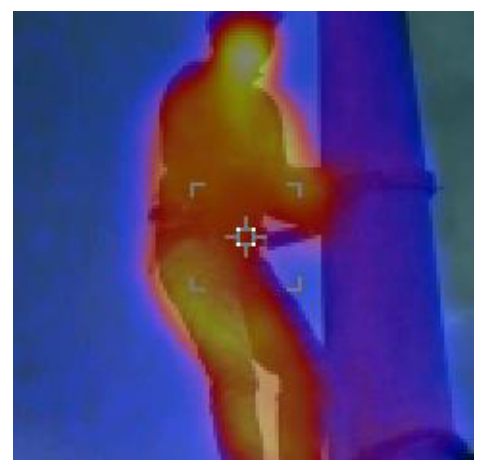

Figure 2. Middle climbing.

The emanation of heat has a radiation on the work clothes. There is a heat radiation from the face that must be discarded.

Concluding the ascent, we find that the muscles graphically present a higher concentration of heat given by the effort required enormously. Figure 3

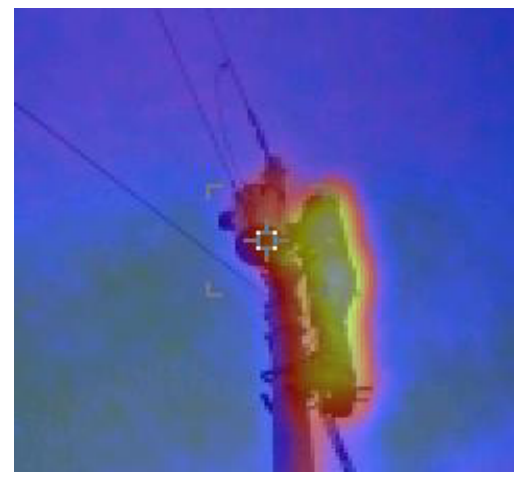

Figure 3. Finish climbing.

\subsection{Control of heart rate increase.}

It was necessary to have a sensor with electrodes to determine the heart rate; the sensor communicated through a cell phone by Bluetooth recording the data in each event of the ascent to the post. Of the electrical linemen studied one has had experience in these types of 
ascent, the other 5 people for the first time carried out these activities.

Figures 1, 2, 3, 4 and 6 show the cardiac pulse curves of novice linemen in these activities.

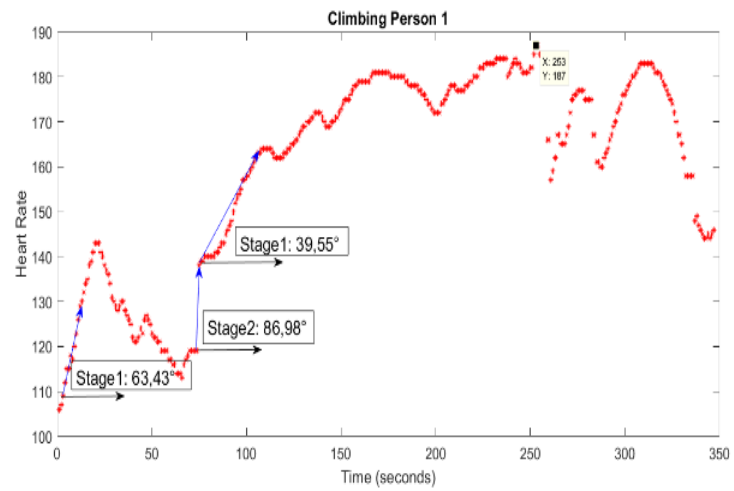

Figure 4. Person 1 Climbing by stages.

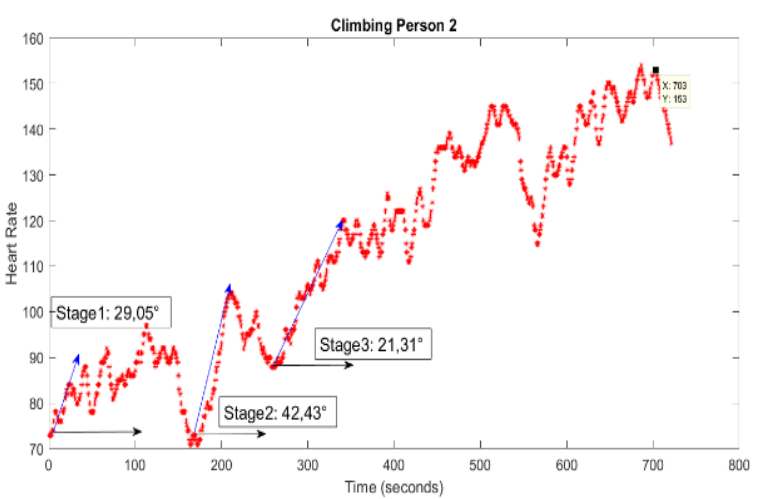

Figure 5. Person 2 Climbing by stages.

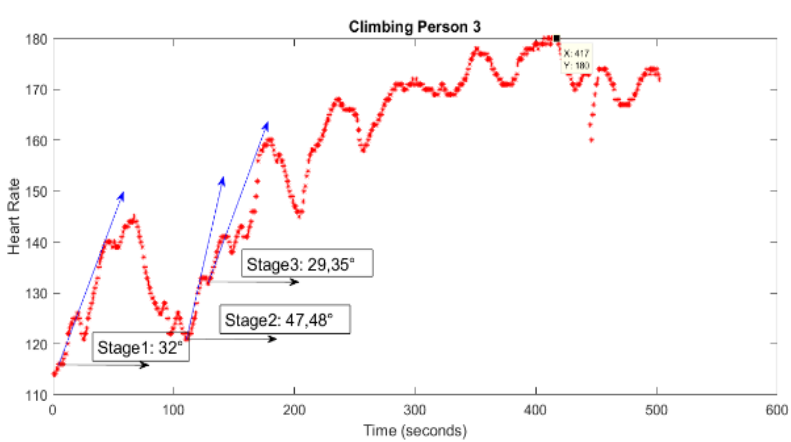

Figure 6. Person 3 Climbing by stages

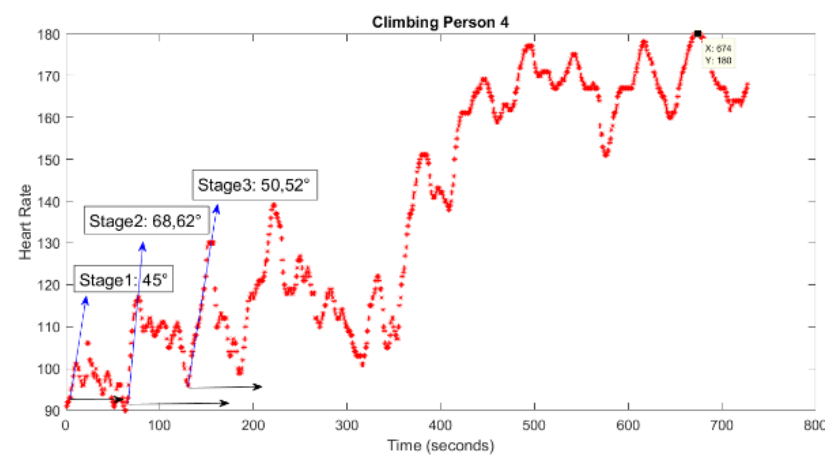

Figure 7. Person 4 Climbing by stages

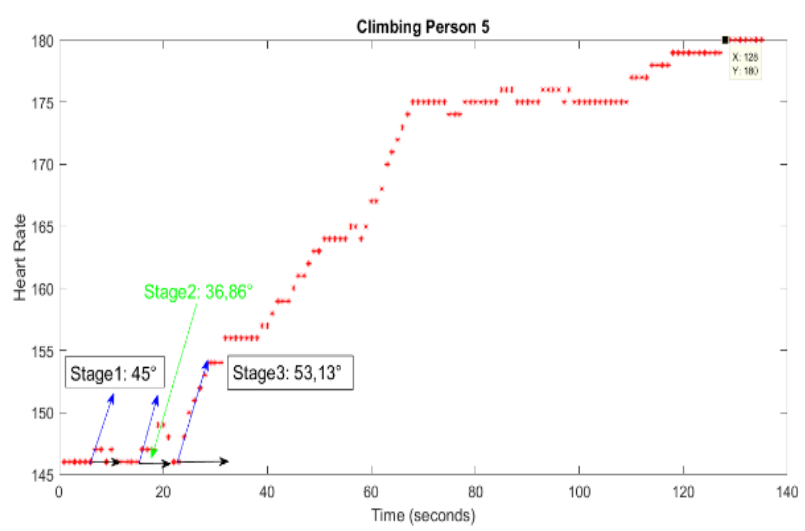

Figure 8. Person 5 Climbing by stages.

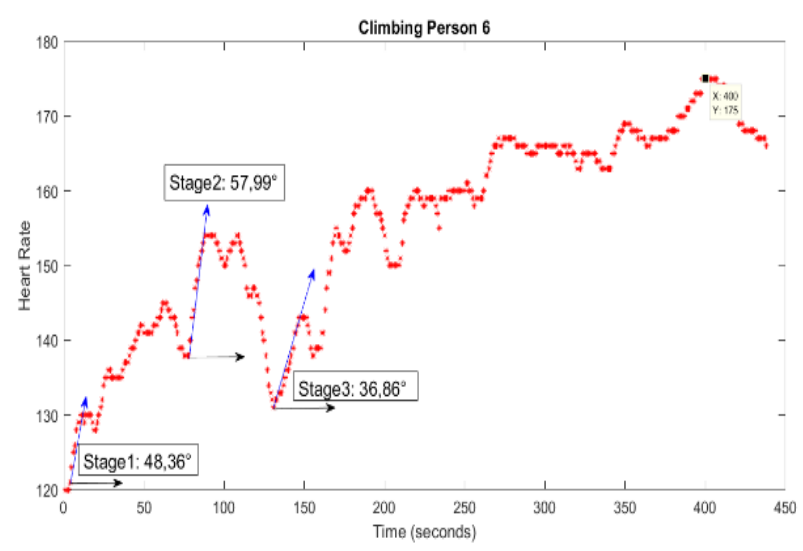

Figure 9. Person 6 Climbing by stages.

\subsection{Analysis of the cardiac pulse in the descent of the electricians}

The workers had an average age of 32 years and with this value the formula so the maximum heart rate calculated is next:

HRmax $=220$-age

HRmax $=220-32=188$

The curves of Figures 10, 11, 12, 13, 14 and 15 were obtained in the ascent and descent of the electric power pole. The maximum allowed heart rate is the blue line.

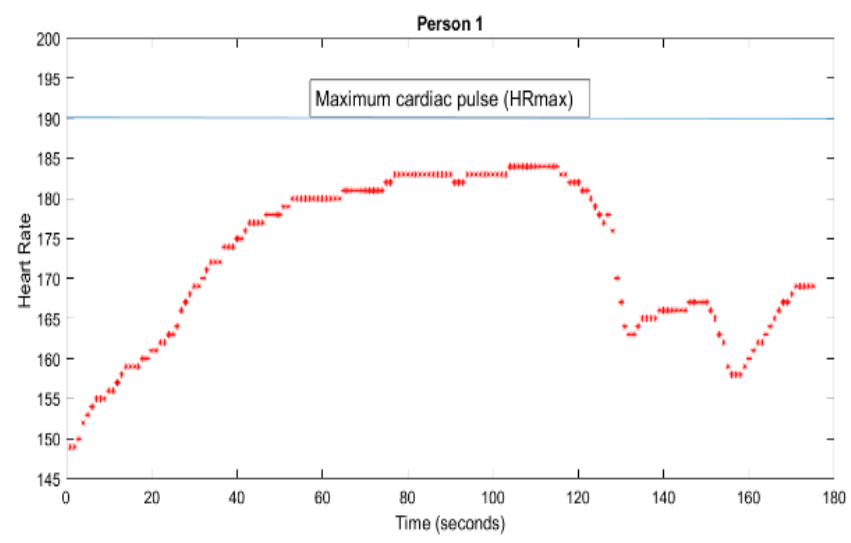

Figure 10. Person 1 Descending. 


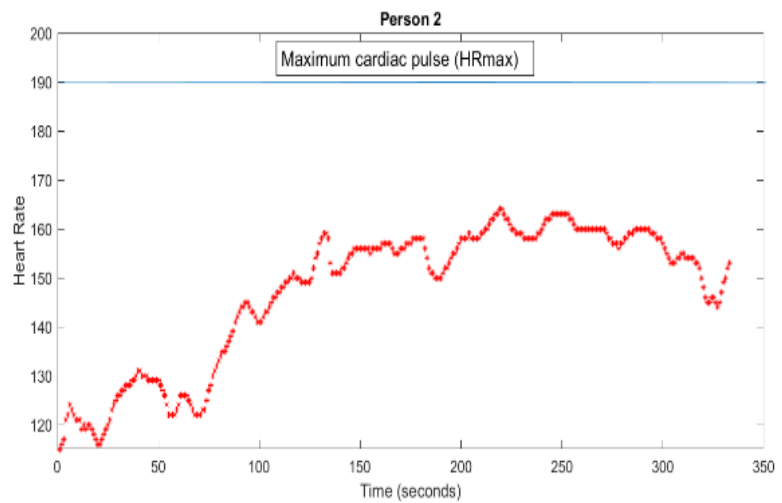

Figure 11. Person 2 Descending

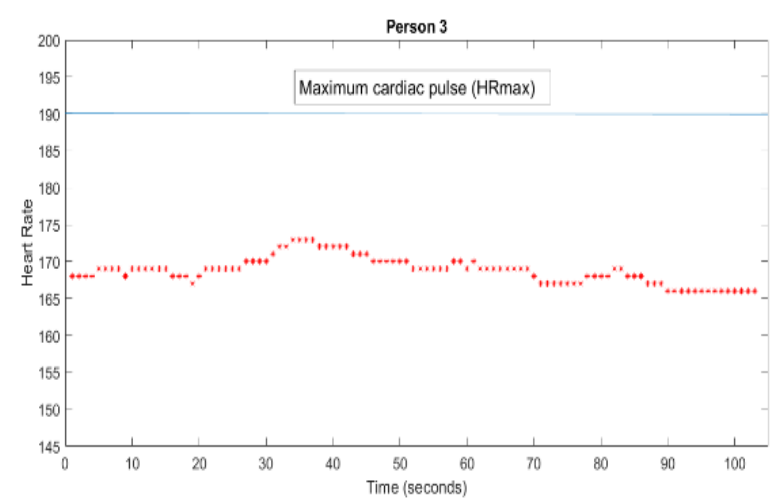

Figure 12. Person 3 Descending.

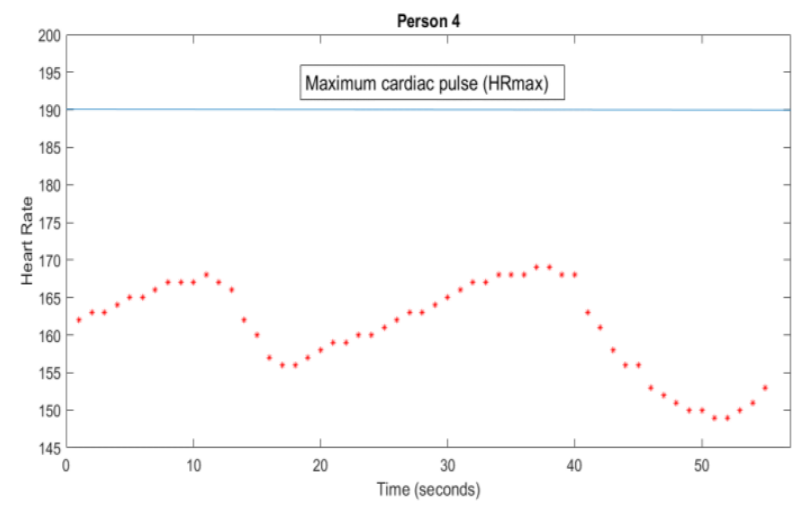

Figure 13. Person 4 Descending.

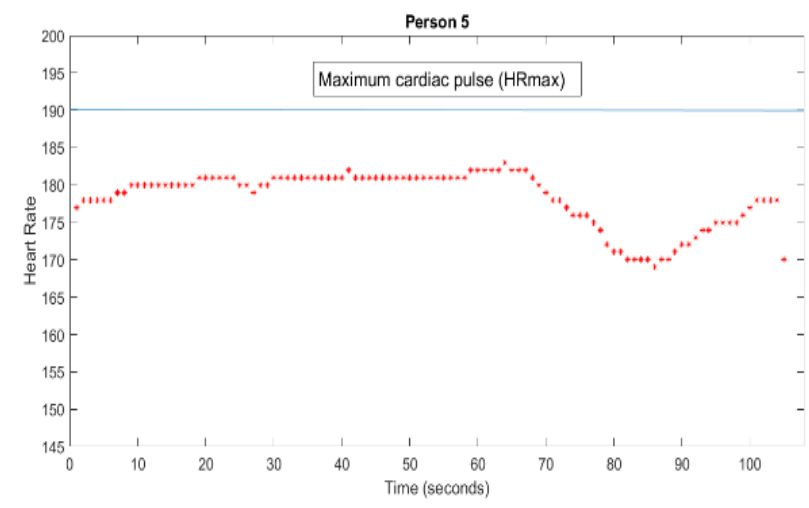

Figure 14. Person 5 Descending.

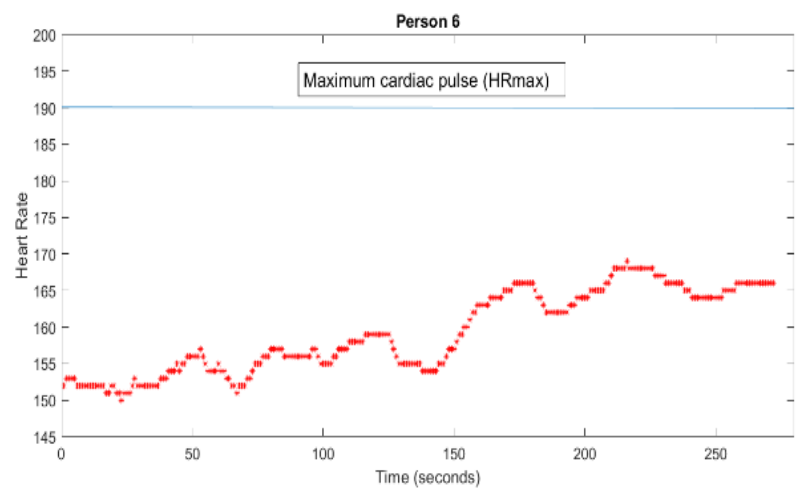

Figure 15. Person 6 Descending.

\section{Analysis of the investigation}

Concordant with the six images, the first step is presented in the column of stage 1 , the second step in stage 2 and the steps to scale all the concrete posts are presented in stage 3 . Table 1 .

For stage 1, the electrician keeps one foot on the ground and the other takes the first step on the pole, reflecting an effort required for this activity. For stage 2, the lineman takes the second step on the concrete post, loses contact with the ground and presents a state of anxiety, because of the fear of falling or slipping on the post. In stage 3 continues with the ascent in the post, constantly presenting fear and effort, which is constantly increasing and tends to reach the limit of heart rate.

Table 1. Lifting and lowering details of liners considering angular pending stages.

\begin{tabular}{|l|c|c|c|}
\cline { 2 - 4 } \multicolumn{1}{c|}{} & \multicolumn{3}{c|}{ Angular slope climbing } \\
\cline { 2 - 4 } \multicolumn{1}{c|}{} & Stage 1 & Stage 2 & Stage 3 \\
\hline Person 1 & $63.43^{\circ}$ & $86,98^{\circ}$ & $39,55^{\circ}$ \\
\hline Person 2 & $29,05^{\circ}$ & $42,43^{\circ}$ & $21,31^{\circ}$ \\
\hline Person 3 & $32^{\circ}$ & $47,48^{\circ}$ & $29,35^{\circ}$ \\
\hline Person 4 & $45^{\circ}$ & $68,62^{\circ}$ & $50,52^{\circ}$ \\
\hline Person 5 & $45^{\circ}$ & $36,86^{\circ}$ & $53,13^{\circ}$ \\
\hline Person 6 & $48,36^{\circ}$ & $57,99^{\circ}$ & $36,86^{\circ}$ \\
\hline
\end{tabular}

Considering Table 1. Of the six people, only fifth has been an expert in these activities, which is evidenced in the second stage with a lower cardiac pulse compared with others analyzed, which for the first time carry out this activity.

The rookie linemen in the second step that they give in their ascent to the post have a greater heart rate, product of the fear to be hurt by a possible fall to the ground.

In the third stage, a continuous and constant increase is maintained for the experts and inexperienced on the rise of posts.

In the descent of the post, cardiac pulse is unpredictable and possibly variable by the different states of mind of the workers, for the fulfillment of their objective that possibly seemed impossible to achieve it. 


\section{Conclusions}

In a person with too much stress, the cardiac pulse tends to increase, so that his heart rate differs in the states that are presented. In the second stage, there is a greater difference in the state of stress and fear. However, in the expert people decreased heart rate, given by an absence of fear and stress.

Due to the different physical state, physiology and mood of the participants, it is difficult to predict the heart rate of the participants when concluding the ascent to the post.

From each one of them there are different forms of curves that can be observed in the extracted graphs of the cardiac impulse measuring equipment.

The attention given to the cardiac pulses of people in different jobs will definitely avoid catastrophic work consequences.

\section{Acknowledgment}

Our sincerely thanks to Universidad Técnica de Cotopaxi and Msc. Diana Marín for collaborating with logistics for the presentation of the present investigation work.

\section{References}

1. M. Tuttle, C. Ozemek, H. Finch, L. Kaminsky y M. Whaley, Am. Heart Ass., Inc., "Quadratic Regression Reveals an Accelerated Age-related Decline of Maximal Heart Rate in those with Low and Moderate Cardiorespiratory Fitness", Available: http://circ.ahajournals.org [Accedy February. 12, (2017).

2. C. A. Fourlas, Eur. Heart Jour. "Resting heart rate and excessive heart rate increase during pre-exercise mental stress: which one predicts mortality?", . 30, 2815 (2009)

3. T. Pulido, J. H. Donis, D. F. Dávila, C. Hernández and R. Odreman, Adv. Biom., "Assessment of heart rate and its relationship with ventricular remodeled in dynamic exercise as indicator of autonomic function between endurance athletes and strength athletes", 5, 57-65 (2016).

4. Gamberale, Jour. Erg. "Perceived Exertion, Heart Rate, Oxygen Uptake and Blood Lactate in Different Work Operations", 15, 545-554 (1972).

5. H. M. Trice and W. J. Sonnenstuhl, The Jour. App. Beh. Sc., "Drinking Behavior and Risk Factors Related to the Work Place: Implications for Research and Prevention", 24, 327-343 (1988). 